\title{
A Burning Desire: \\ Steps Toward an Evolutionary Psychology of Fire Learning
}

Daniel M. T. Fessler* **

\begin{abstract}
Although fire is inherently dangerous, leading many animals to avoid it, for most of human history, mastery of fire has been critical to survival. Humans can therefore be expected to possess evolved psychological mechanisms dedicated to controlling fire. Because techniques for starting, maintaining, and using fire differ across ecosystems, the postulated adaptations can be expected to take the form of domain-specific learning mechanisms rather than fixed behavioral templates. After outlining features that such mechanisms are predicted to possess, I review the literature on fire play in western children, finding that attraction to and interest in fire is widespread, experimentation with fire often begins in early childhood, and fire play typically peaks in late childhood or early adolescence. The latter aspect stands in contrast to results from a survey of ethnographers which reveals that, in societies in which fire is routinely used as a tool, children typically master control of fire by middle childhood, at which point interest in fire is already declining. This suggests that fire learning is retarded in western children, arguably due to patterns of fire use in modern societies that are atypical when viewed from a broader cross-cultural perspective. Together with the fact that western entertainment media provide a distorted portrait of the properties of fire, this pattern, while limiting the value of naturalistic observations of fire learning in the West, nevertheless has the benefit of providing a strong testing ground for future experiments exploring the universality of the psychology underlying the control of fire.
\end{abstract}

\section{KEYWORDS}

Fire, children, learning, fire play

* Center for Behavior, Evolution \& Culture and Department of Anthropology, 341 Haines Hall, UCLA, Los Angeles, CA 90095-1553, U.S.A. Tel: +1 (310) 794-9252, Fax: +1 (310) 206-7833, Email: dfessler@anthro.ucla.edu

** Acknowledgements: I am grateful to the many investigators, listed beneath Table 1, who contributed ethnographic observations for this paper. 


\section{Introduction}

The use of fire is a human universal, a behavior present in virtually all societies (Brown, 1991). Indeed, while there is debate as to the earliest evidence for the controlled use of fire, evolutionary anthropologists trace the manipulation of fire far beyond the origins of our species - with suggestive findings dated as early as 790,000 years ago (Goren-Inbar et al., 2004), and some even surpassing the one million year mark (Brain \& Sillen, 1988), it is clear that the history of hominids' exploitation of fire is quite deep. The utility of fire for our hunter-gatherer ancestors and their predecessor species was likely multiplex. In addition to fire's obvious benefits as a source of warmth and light, fire played a key role in the evolution of the human diet, as cooking would have allowed our species to exploit a vastly expanded array of plant and animal foods (Ragir, 2000; Stanford, 1999; Wrangham, Jones, Laden, Pilbeam, \& Conklin-Brittain, 1999). Likewise, the control of fire allowed for applications in hunting, combating predators and hostile conspecifics, managing wild plant resources, and tool production (cf. Clark \& Harris, 1985). Of course, at no point in our species' history will fire have constituted an unadulterated good - together with the smoke that it produces, fire is a significant source of injury and death, a pattern reflected in most animals' wariness of fire.

With a deep evolutionary history and a profound impact on fitness, there is every reason to believe that fire has been a source of recurrent and substantial selective pressures shaping human behavior and the psychological architecture that underlies it. However, despite the uncontroversial, perhaps incontrovertible, nature of this conclusion, to date, evolutionary psychologists have largely ignored fire's role in human evolution. The purpose of this paper is to call attention to this lacuna, and suggest some possible solutions.

The human use of fire can be broken down into two elementary components, namely controlling fire and producing fire. Fire occurs naturally due to lightning strikes, volcanic activity, and concentrated solar radiation. In contrast, the artificial production of fire requires highly specialized tools, techniques, and materials. While this suggests that early hominids acquired the ability to control fire long before they were able to produce it, importantly, all available evidence indicates that, like the 
production of fire, the control of fire is a learned behavior that is profoundly contingent on experience. This observation merits further consideration. By way of contrast, consider the manufacture of the spider's web or the salmon's nest. While each involves relatively complex constructive behavior that employs variable features of the environment, in light of the life cycles of these organisms, these activities are necessarily governed by innate templates rather than acquired information. Given a) the chronological depth of the hominid relationship with fire, b) the benefits of controlling fire, and c) the costs of erring in this enterprise, this raises the question as to why natural selection did not similarly favor the evolution of largely innate fire-management skills in humans. One possible explanation is that, unlike spiders or salmon, humans and their predecessors occupied a wide range of ecosystems.

Many of the environments occupied by humans are characterized by enormous variety in the features relevant to controlling fire, principal among which is the nature of available fuels. Combustibles vary with regard to a number of properties relevant to controlling fire, including ease of ignition, rapidity of combustion, and explosiveness. Accordingly, had natural selection favored the evolution of a template or schema akin to those that appear to govern some animal constructions (i.e., the patterned use of materials, having differing specified properties, in a set of discrete tasks characterized by a fixed temporal sequence), such a template would have been of limited utility, as it would necessarily have been parochial to a given ecosystem due to its reliance on fixed types rather than attributes. This is because, unlike, for example, the twigs and feathers used to construct a bird's nest, many of the properties of combustibles are not detectable on the basis of surface features - examining downed tree limbs, for example, will not reveal the features of combustion typical of each type of wood, to say nothing of inspecting materials such as animal fat, beeswax, or tar. Because the techniques involved in building and maintaining a fire are largely dependent on the fuels selected, any pre-existing template for such techniques would have had to specify the fuels to be employed. Given that the only way that natural selection could have produced a fire-management template would thus have been to specify fixed ingredients (akin, for example, to the beaver's fondness for willows, cottonwood, and aspen), and given that such a template would therefore have failed to translate effectively across 
ecosystems, the only avenue open to selection processes operating on a species as wide-ranging as ourselves was to rely on learning for the acquisition of the requisite behaviors.

Importantly, contrary to portraits common in the conventional social science literature, a reliance on acquired information in no way indicates the absence of domain-specific evolved psychological mechanisms (see Tooby, Cosmides, \& Barrett, 2005). On the contrary, beginning with Garcia, Ervin, and Koelling's (1966) seminal demonstration of specialpurpose mechanisms dedicated to the acquisition of toxin-avoidance behaviors, evolutionists have embraced a perspective wherein psychological adaptations can operate via a predisposition to attend to discrete cues and particular sources of information in order to learn about specific facets of the environment. Well-documented examples include snake detection in primates and humans (Mineka, Davidson, Cook, \& Keir, 1984; Öhman, 1993) and inbreeding-relevant kin recognition in humans (Wolf, 1993).

\section{Expected properties of a mechanism dedicated to fire learning}

In exploring the properties that we might expect to characterize the information-acquisition mechanisms specific to the problem of controlling fire, it is useful to begin by considering the logical features of the task domain. Parallels between this domain and that of another, betterstudied, information-acquisition task can shed light on these features. For at least two reasons, the task domain of learning to control fire is akin to that of learning to cope with threats posed by predatory animals. First, paralleling the problem of identifying and differentiating among fuels, although some cues are shared by many predators (e.g., large, sharp teeth), these cues are not universal and, moreover, the color, size, morphology, and behavior of predators varies widely across ecosystems, making it difficult for natural selection to imbue human minds with innate predator-recognition templates (Barrett, 2005). Second, generalized, feedback-based learning is inappropriate to the task of acquiring information about predators since, particularly for children, the costs of such learning would be prohibitively high - as Barrett observes, "A child 
that requires attack or injury to learn that an animal is dangerous is unlikely to survive for long," (2005:217). Correspondingly, if children were to acquire their knowledge of fire exclusively through individual trial-and-error learning, a large percentage would suffer serious or fatal burns.

Barrett (2005) outlines five features that can be expected to characterize the information acquisition system dedicated to learning about predators; we can expect parallels to exist for each of these features in the domain of controlling fire. First, learning can be expected to occur without extrinsic motivation. Just as dangerous animals appear to hold considerable fascination for children, so too can children be expected to be spontaneously interested in fire - children are predicted to be curious about the flammability of various materials, and interested in the consequences of manipulating or rearranging burning objects, adding objects to or removing objects from a fire, and so on. Second, we can expect information to be acquired rapidly, often on the basis of only a single exposure. To acquire a new animal concept, children are expected to need only a single encounter, or to hear only once about the attributes of a dangerous animal. Likewise, fire learning should be rapid in response to minimal exposure - information relevant to the control of fire is predicted to have high salience such that children will attend to this information and recall a greater percentage of it following exposure than will be the case with respect to information, relevant to other domains, that is acquired through general-purpose learning mechanisms. Third, both because it allows low-cost learning-at-a-distance and because it tracks local ecological circumstances, socially-transmitted information constitutes a valuable resource for both the student of predators and the student of fire. Just as members of a local group act as a reservoir of accumulated observations regarding the identifying attributes and characteristic behaviors of indigenous animals, so too can others' knowledge of the fire-relevant properties of locally-available materials be expected to play a valuable role in learning to use fire in a given ecosystem. Fourth, the information acquisition system can be expected to employ biases or prior structures that guide learning in the specified domain. For example, Barrett suggests that children's learning about animals may be guided by such fundamental categorical distinctions as dangerous versus harmless, carnivore versus herbivore, and so on. Correspondingly, 
learning about fire may be structured by dichotomies such as flammable versus nonflammable or, more broadly, things that intensify fires and things that diminish fires. Lastly, acquisition of procedural knowledge may be achieved in part through the operation of dedicated mechanisms that serve to generate relevant experience in a safe context. Just as chase play, an intrinsically rewarding activity for children, may serve to hone predator-prey pursuit and evasion skills (Steen \& Owens, 2001), so too may children's manipulations of small fires, embers, and the like provide a means of acquiring relevant experience in a setting that is safer than a full-scale cooking or heating fire.

\section{Existing literature: Children's fire play and fire setting in modern societies}

Testing the five sets of predictions described above will require structured experiments involving young children from diverse cultures. Because such an enterprise is not entered into lightly, it is worth exploring here the extent to which available findings are congruent with the portrait of human fire psychology outlined above. A voluminous literature addresses the topic of fire setting by children and juveniles. Importantly, fire setting is a broad category of behavior, encompassing everything from playing with matches to arson. The perspective developed here suggests that, for the child, observation of, and interaction with, fire will often be ends in themselves, a motivational orientation that differs markedly from that which pertains when fire is used as a weapon to intentionally inflict damage or harm. Consistent with this distinction, public safety experts distinguish so-called 'fire play' from other behaviors involving fire, defining fire play as "A fire deliberately set for no purpose, constructive or destructive, beyond the fire itself," (Hall, 2003:1). This distinction, however, in no way suggests that fire play is harmless - on the contrary, fire safety officers' concern with fire play is quite justified. By way of example, in the U.S., between 1980 and 1999, children's fire play resulted in 7,306 civilian deaths, 48,922 serious civilian injuries, and over 3 billion dollars' worth of property damage (Hall, 2003). Although governmental attempts to combat the problem through consumer product safety regulations, public information campaigns, education plans, and interven- 
tion programs have succeeded in reducing these figures somewhat, children's fire play continues to be a significant source of pain, suffering, and expense in modern societies.

Much of the literature on fire setting is aimed at characterizing the features of recidivist juvenile fire setters, with investigators identifying attributes and correlates such as cruelty to animals, defiance of authority, and a chaotic or conflictual family environment as risk factors for recidivism (Kolko, 2002). While such research usefully identifies dangerous individuals who are relatively resistent to the mechanisms of social control normally employed to enhance conformity to social standards, this entreprise generally overshadows the exploration of fire setting among non-clinical, non-recidivist children. Nevertheless, despite this bias, it is possible to glean a portrait of such behavior from the existing literature.

Kafry (1980) queried 99 lower elementary school boys in Berkeley, California, finding that $45 \%$ reported having played with fire, and $21 \%$ reported having caused a fire as a consequence; of the latter, 18\% were caused by children who were two years of age or younger at the time of the accident. Eighty-one percent of children surveyed reported being interested in fire and, across ages, curiosity was the motive cited most frequently to explain the behavior. As a comparison group for a clinical sample, Kolko et al. (2001) conducted a three-year longitudinal study of a community sample of 100 boys and 62 girls, age 6-13 (mean = 9.5), with follow-up interviews at one and two years. Results reveal quite consistent patterns wherein, at any one time, approximately one-third of the children report being interested in fire, slightly less than half report playing with matches, and slightly less than one-third report having set play fires. Viewed individually, by the end of the study almost threequarters of the participants had at some time played with matches or set a play fire. Perrin-Wallqvist and Norlander (2003) retrospectively surveyed 50 18-year old Swedish men and 45 18-19 year old Swedish women. Seventy percent of the men reported having played with fire during childhood, and $44 \%$ of the women did likewise. The principal motives for childhood fire play were curiosity, entertainment, and excitement seeking; a high proportion of subjects reported being fascinated or drawn to fire. Paralleling this result, Perrin-Wallqvist et al. (PerrinWallqvist, Archer, \& Norlander, 2004) surveyed 28 male and 28 female 
Swedish 16-year olds concerning emotional attitudes toward fire. While $61 \%$ thought fire was "cozy" and 20\% found fire "exciting," only $9 \%$ thought that fire was "uninteresting"; $27 \%$ of the respondents found fire sufficiently entertaining that they lit fires when bored.

Simonsen and Bullis (2001) surveyed 5,416 third through eighth grade Oregonian students, relatively evenly distributed across ages, regarding fire starting, interest in fire, and motivations for fire play. Forty-seven percent of students reported having played with fire; a retrospective question revealed that $10 \%$ reported starting their first fire between the ages of one and five, $27 \%$ reported starting their first fire between the ages of six and ten, and 10\% reported starting their first fire between the ages of 11 and 15. Attraction to and interest in fire was quite high: although only $29 \%$ overtly reported being interested in fire, $46 \%$ reported liking television programs that involve fire, $61 \%$ reported enjoying assisting adults in lighting or putting out fire, 33\% reported enjoying burning objects such as twigs, grass, or paper, and 21\% reported enjoying playing with matches or lighters. Correspondingly, fire had high entertainment value for children, with large proportions of respondents citing as motives for fire starting "just for fun" $(23 \%)$ and "out of boredom" $(19 \%)$, with curiousity ("to see what would happen") another significant contributor $(18 \%)$.

Cotterall (2003) conducted a similar survey of 3,031 students from grade 3 through 13 in Sudbury, Ontario. A number of patterns are markedly evident. First, while the frequency of actual fire play shows a steady increase across the age span studied (18\% of third graders report having played with matches or lighters in the last year, while $81 \%$ of thirteenth graders report having done so), what Cotterall terms 'expressed preoccupation and fascination with fire' (e.g., "Are you curious about fire?" "Are you really interested in fire and what it can do?" etc.) does not vary in any regular fashion across this age range and, likewise, the lower-level factor that Cotterall terms 'expressed interest in fire' (e.g., "Do you like to start fires?" "Do you feel excited when you watch a fire burning?" "Do you like staring at fire?" etc.) shows only a gradual increase with age. These patterns suggest that the age-related changes in fire play documented by Cotterall are a function not of changes in attraction to fire, but rather of changes in willingness to disobey rules, changes in opportunities to engage in proscribed behavior outside of 
adult supervision, and changes in access to sources of ignition. Tellingly, across ages, the principal motives for fire starting are "just for fun," "to see what would happen," "to destroy something," and "boredom," patterns that are even more marked among those who reported extensive fire play. Equally important, knowledge regarding institutionally-conveyed fire safety practices had virtually no relationship with the extent of fire play.

An indirect measure of children's fire-related activity can be derived from records of fires that, whether by design or accident, become sufficiently large as to attract the attention of fire departments. Okulitch and Pinsonneault (2002) compiled records of 4,600 fires involving juveniles in Oregon between 1996 and 2000. Twelve-year olds accounted for the largest proportion of these fires, with a steady increase in involvement from ages 1 to 12 , and a steady decrease in involvement after age 12. If we assume that, in at least some sizable fraction of these fires, the fire starter had no intention of creating a conflagration sufficiently large as to result in the involvement of the authorities (cf. Kafry, 1980), then, comparing this pattern to Cotterall's findings suggests that, in North America, age 12 constitutes the dangerous apex of the combination of factors listed above (willingness to disobey rules, etc.) and incomplete mastery of fire, such that it escapes the fire starter's control.

Lastly, although not presented in the context of quantitative data, drawing on her experience with firesetters in Massachusetts, Pinsonneault concludes that "[c]ommon questions children have about fire include, 'What makes fire hot? How does a small fire grow? .. . Why are some fires very smoky? . . C Can everything burn? . . How can you keep a fire small? How can you put fires out?" (Pinsonneault, 2002:223). Importantly, these are exactly the sort of questions that we might expect to be foremost in the minds of individuals whose curiousity is not simply the product of a general interest in the world, but rather reflects a domain-specific information-gathering system the purpose of which is to acquire the ability to control fire.

Although the studies reviewed above vary considerably in their details, they can be roughly summarized as follows: a) western children first become curious about fire in early childhood, b) experimentation with fire increases as a function of age, often extending well into adolescence, and c) for many individuals, some attraction to fire persists into maturity, 
a pattern consistent with the entertainment value that many western adults place on activities such as gazing into a fire. The question remains, however, as to whether this trajectory directly reflects the workings of a developmental information acquisition system governed by an evolved psychological mechanism. In particular, it is telling that much fire play occurs in adolescence in western populations. First, as was arguably true in most ancestral human societies, in many small-scale societies today, adolescents, particularly females, engage in a great deal of productive unsupervised economic activity (cf. Kaplan, Hill, Lancaster, \& Hurtado, 2000). Given that fire is an elementary part of the human tool kit, it would be odd if the acquisition of fire-control skills was normally so retarded that additional experimentation was still required during the same phase of life when significant economic productivity has already commenced. Second, a hallmark of adolescence in western nations is a substantial increase in mobility and autonomy, changes that often precipitate engagement in proscribed activities that had previously been precluded by caretaker supervision. Combined with strong prohibitions against fire play and, for many, limited exposure to fire on a daily basis, this pattern means that, in much of the developed world, adolescence often constitutes the first opportunity to extensively experiment with fire. Together, these observations suggest that, while studies of fire play in the West are interesting in as much as they reveal considerable motivation to explore fire from a young age, they nevertheless present what is likely a highly distorted portrait of the developmental unfolding of a mechanism aimed at acquiring fire knowledge and skills.

\section{Children's interactions with fire in societies in which fire is routinely used as a tool}

\section{Children and fire in Bengkulu, Sumatra}

Between 1990 and 1993 I conducted ethnographic fieldwork in a semitraditional Bengkulu Malay fishing village on the west coast of Sumatra. Although I did not systematically collect data on children's interactions with fire, my immersion in village life was such that I nevertheless recorded many offhand observations and impressions, allowing for a sketch of children's interactions with fire. 
Because childcare duties and cooking duties were often delegated to the same individuals, from infancy, on children in Bengkulu had ample opportunity to observe others controlling fire. Small children often sat on or near their caretakers while the latter manipulated fire; caretakers brushed the child aside with a scolding if the child reached for burning or hot items, but otherwise offered no instruction regarding the control of fire. Children's autonomous manipulation of fire typically began around age 4. About this time children were allowed to build small fires, and frequently did, cooking either mud pies and other pretend foods or actual morsels. Adults did not instruct children with regard to either building or maintaining a fire, at most snapping at a child if play with or near fire posed a danger; the only input that might be considered pedagogical came from slightly older children, who would sometimes narrate their actions using a type of play-speech while handling fire. Children's interest in such "kid's fires" seemed to peak around age 6-7 and diminished rapidly thereafter, disappearing entirely before age 10. Children ages 3-6 sometimes poked at, jumped over, and similarly toyed with adult fires (resulting in one serious burn during my stay), but this was limited in scope and frequency, and, again, had largely subsided by middle childhood. Children age 6 and up were often sent to a neighbor's house to fetch burning embers for fire-starting. By age 8 both girls and boys were commonly performing adult chores, including cooking and tending fires, and their interactions with fire were increasingly utilitarian in nature. Adolescents and young adults (particularly men, nearly all of whom smoked) would occasionally idlely flick cigarrette lighters, toy with matches, or poke at burning candles, but such behavior was more akin to fiddling than to interested experimentation, and was not characterized by any excitement. The only behavior that I observed that could even remotely be classified as fascination with fire occurred when a fad for making cap bombs swept the village; boys age 5-8 would modify old spark plugs to form the body of the bomb, scrape match heads to obtain gunpowder, then load the projectiles and throw them upward, squeeling with glee when they produced a resounding Bang! upon impact with the ground.

To summarize my (admittedly unsystematic) ethnographic observations: in one Bengkulu village, children experienced early interaction with fire but received little or no instruction in controlling it. Typically, children 
had gained complete mastery of fire by age 7 and, for most older children, adolescents, and adults, fire was of only passing interest. To explore whether this pattern might be representative of societies that use fire in ways more akin to those likely characteristic of ancestral humans, I surveyed other ethnographers with regard to their recollections about children's interest in, play with, and mastery of fire.

\section{Ethnographers' impressions from 19 societies}

I sent a questionnaire covering six general areas of interaction with fire (see Appendix) to anthropologists and others with extensive ethnographic field experience. Eighteen investigators responded, providing information on 19 cultures; Table 1 summarizes this information. None of the investigators had conducted research focusing directly on children's interactions with fire, hence, like my own observations from Bengkulu, their responses should be considered preliminary accounts of this topic, rather than definitive portraits thereof.

Though not evenly distributed across extant traditional and semitraditional societies, the survey results do capture disparate geographic regions, suggesting that any resulting patterns are unlikely to be explicable as merely the product of cultural sharing or cultural diffusion. Although variation exists, several themes stand out. First, children begin interacting with fire early, with ages ranging from toddlerhood to 6 . Second, children generally do not play with fire in the manner characteristic of western children: in most cultures studied, there is little or no play behavior involving fire and, when it does occur, such behavior seems to have practice or semi-utilitarian functions akin to those that I observed among the Bengkulu - with the exception of several reports of interest in firecrackers or their homemade equivalents, none of the reports describe children igniting materials simply to watch them burn. Consistent with these observations, the majority of respondents report that fire has no entertainment value whatsoever - fire is viewed in strictly utilitarian terms, and fire-gazing generally does not occur among children or adults. Reports of children's fire competence vary somewhat; most of the respondents were more confident describing children's ability to tend or maintain a fire than they were reporting on children's ability to build a fire, in part because, in many of the societies studied, people rarely start a 
fire from scratch, relying instead on embers or coals from another fire. Gender differences with regard to fire competence were described for a number of the societies, with girls generally acquiring competence sooner than boys and/or achieving greater proficiency due to a gendered division of labor. Focusing on whichever gender achieves proficiency earlier, the age for achieving competence in maintaining a fire ranges from 5 to 8 , with the average being approximately 6.5 .

\section{Discussion}

To summarize the above, while some variation exists, ethnographers working in disparate societies in which fire is routinely used as a tool report patterns of fire behavior similar to those that I observed in Sumatra. Children's interactions with fire begin early, with mastery of fire typically achieved by middle childhood. Fire is viewed in utilitarian terms it has little or no entertainment value, and children's fire play, when it occurs at all, is best described in terms of practicing a necessary skill. These patterns underline the contrast between the developmental trajectory of fire learning in modern societies and that which occurs elsewhere. For ancestral humans and their immediate predecessor species, fire would have been a nearly-omnipresent tool, an important but mundane facet of daily life, and the same seems to characterize people's interactions with fire in many contemporary traditional or small-scale societies. This stands in contrast to fire's role in most modern societies, where it is used principally as decoration (hearth fires, candles), entertainment (bonfires, fireworks), or romantized marker of special circumstances (barbeques, campfires), functions that are neither frequent nor mundane. At the same time that fire is culturally marked with heightened affect, children in modern societies have limited opportunities to learn about fire through such low-level hands-on manipulations as tending a cooking fire or building a play version thereof. Hence, many modern societies may simultaneously enshrine fire as an emotional symbol and a source of amusement (rather than a tool) and constrain children's opportunities to acquire realistic information about, and experience with, fire. One possible consequence of this pattern is that the developmental trajectory of fire learning is greatly retarded in the west, with mastery 


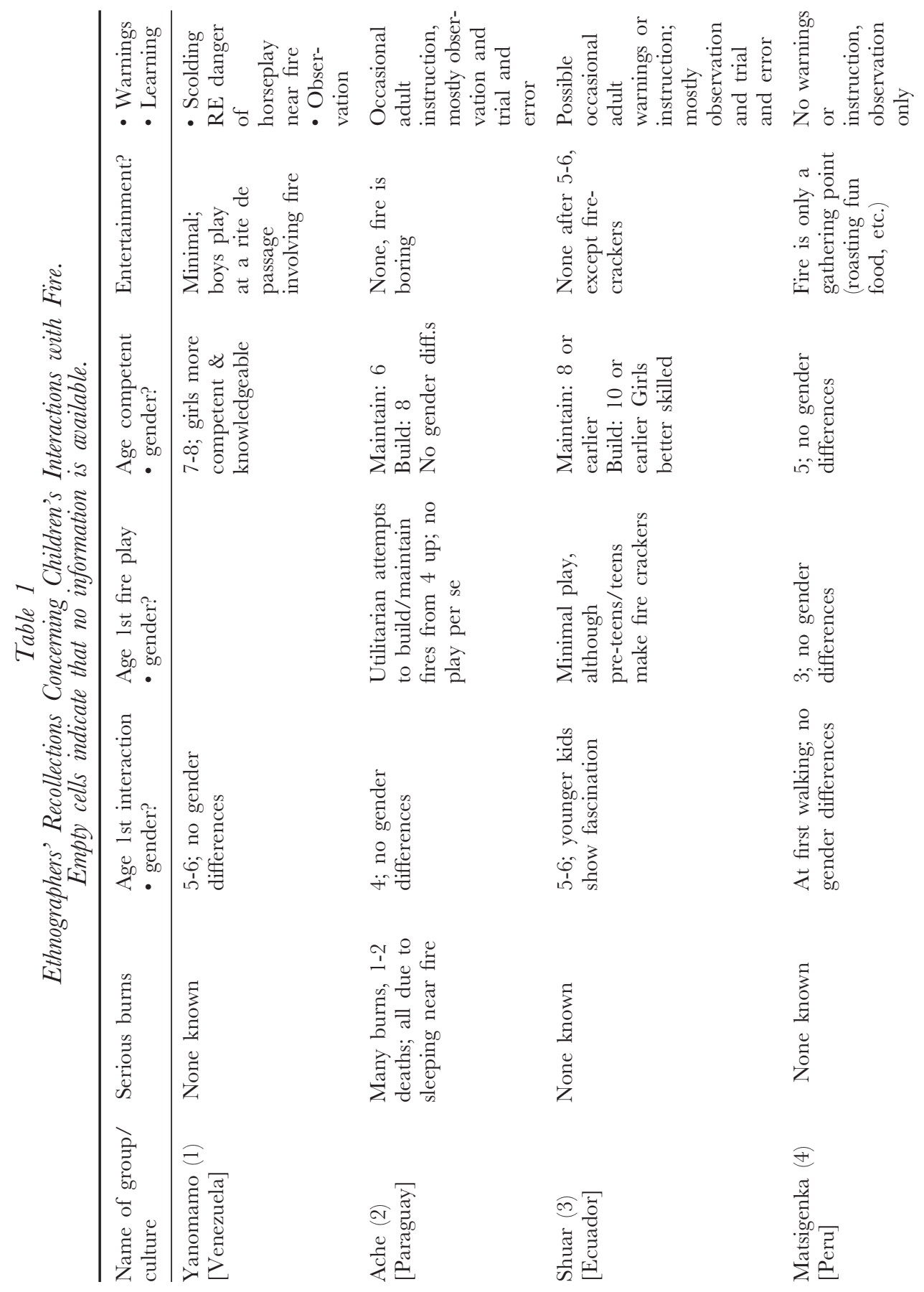




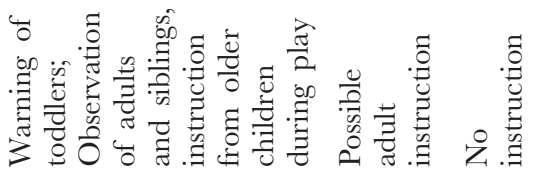

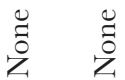

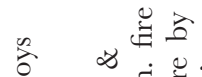

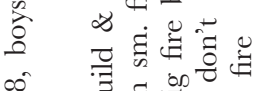

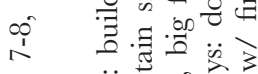

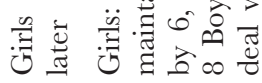

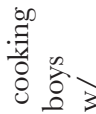

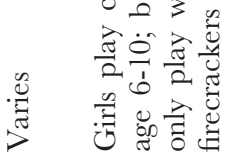

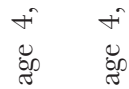

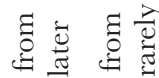

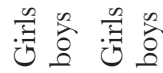

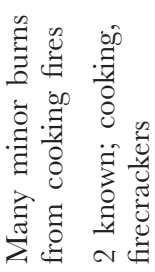

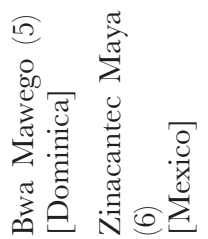

愿

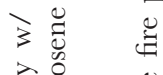

遂总

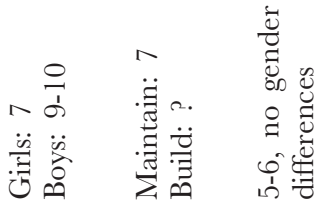

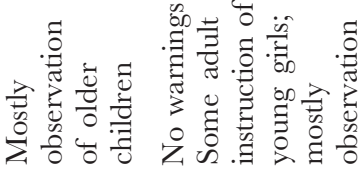

广 胥

을

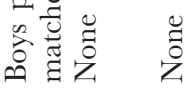

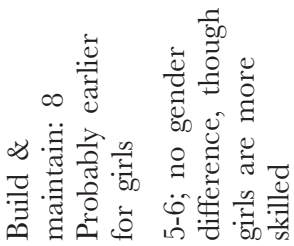

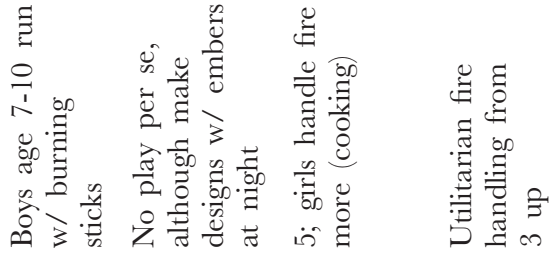

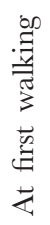

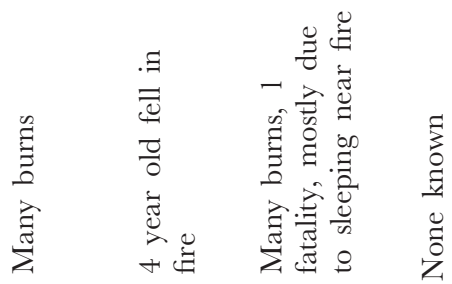

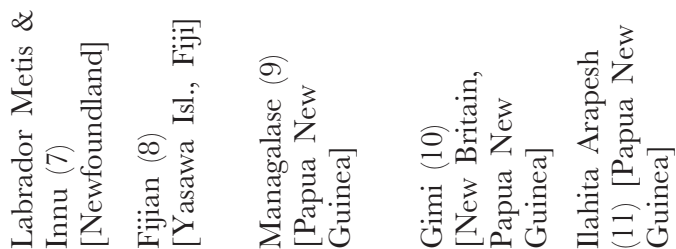




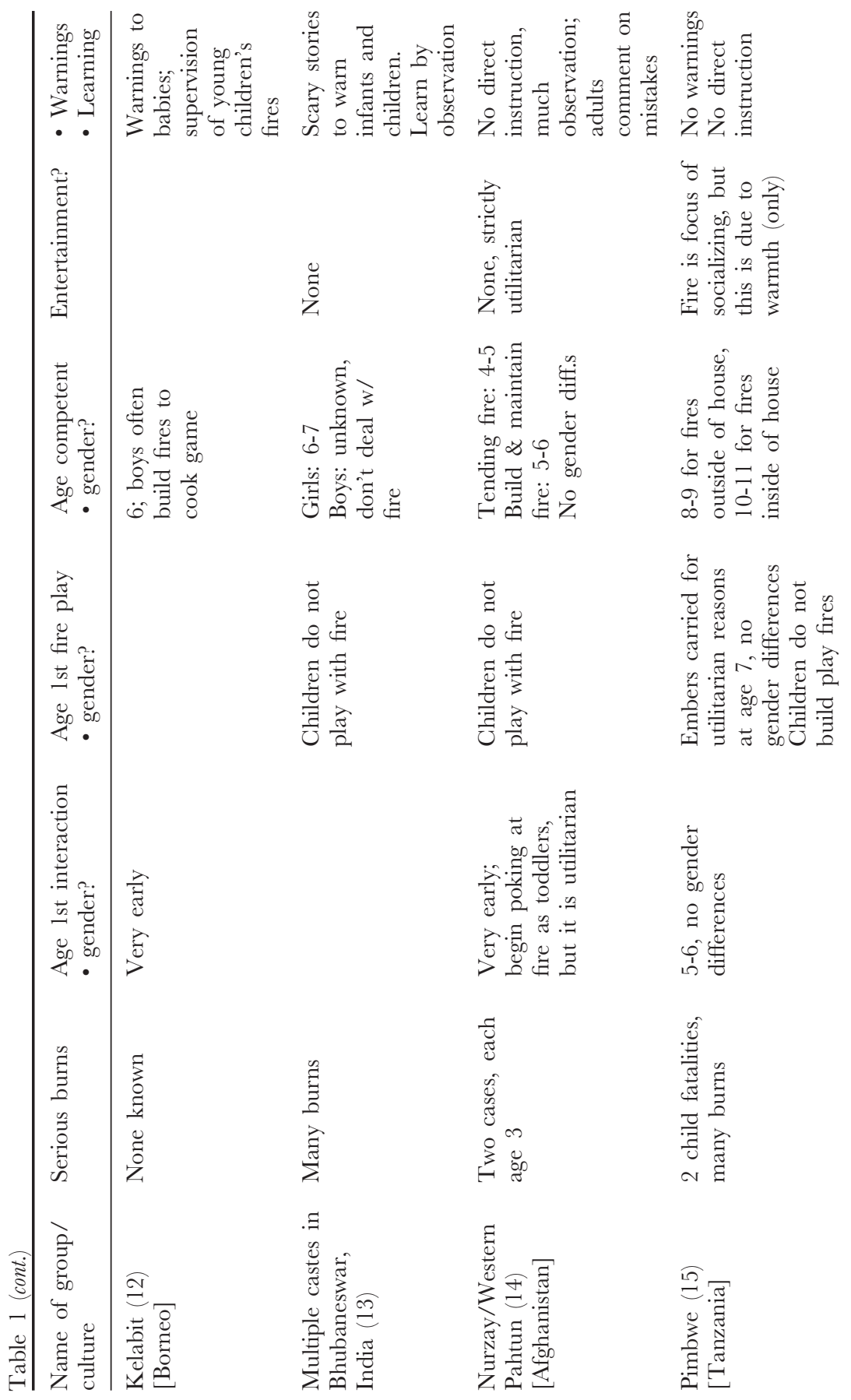




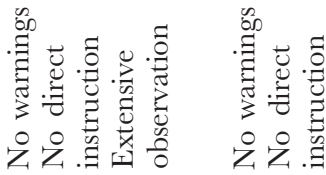
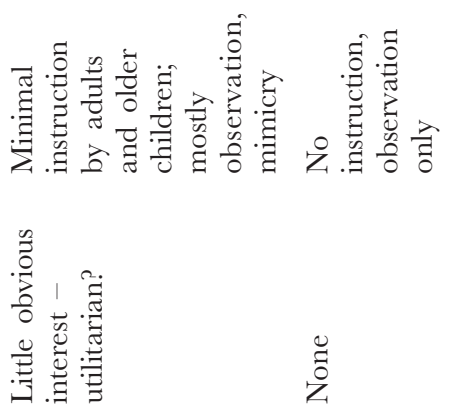

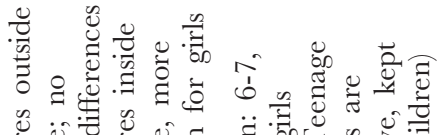

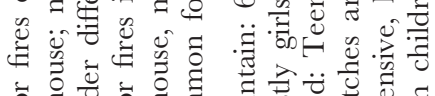

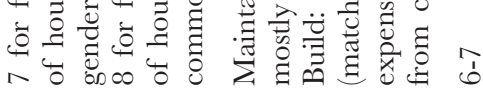

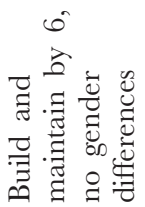

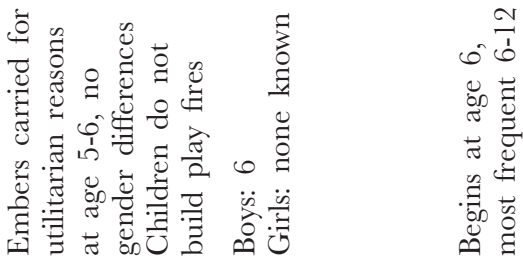

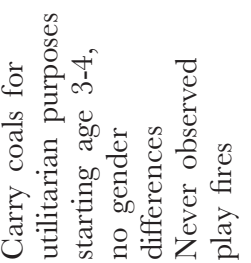

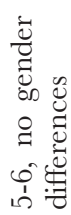
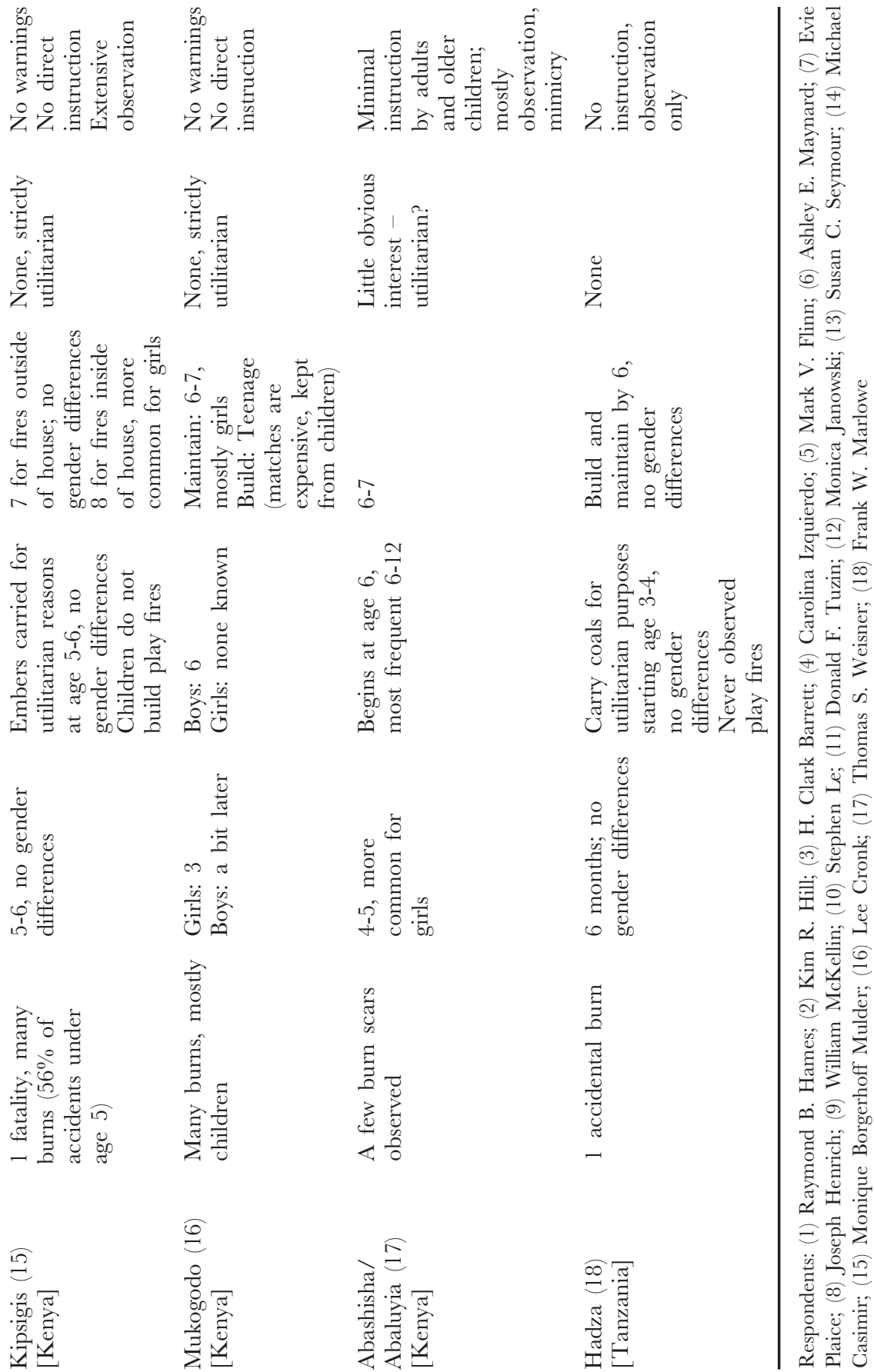
of fire occuring a decade or more later than is the case when children interact with fire in a manner more akin to that which has likely been typical for most of human history. A second possible consequence is that the motives that drive fire learning are only incompletely satisfied, with the result that, throughout life, fire retains greater allure or fascination than would normally be the case; in turn, this pattern provides a personal foundation for the acquisition and perpetuation of the 'toy' meanings ascribed to fire in modern societies. ${ }^{1}$

For scholars seeking to explore evolutionary hypotheses, the possibilities detailed above constrain the utility of some avenues for exploring fire learning in the west, as conclusions derived from naturalistic observations of western children's fire behavior will likely fail to generalize beyond developed societies. However, precisely the same features may also enhance the empirical weight of findings obtained through experimentation. By way of analogy, Barrett and Behne (2005) conducted experiments showing that there are remarkable parallels between German and Shuar children in the development of an understanding of death, parallels that occur despite the fact that German children have far less exposure to actual instances of death, and have far more exposure to misinformation (e.g., cartoon portrayals of immediate recovery from catastrophic injury, etc.). Children in the U.S. are exposed to a plethora of misinformation concerning fire (Hardesty \& Gayton, 2002), including numerous highly unrealistic portrayals of fire on toy packaging (Curri et al., 2003) and in television advertisements (Palmieri et al., 2004). Consider, for example, the lessons that a child might derive from a recent Taco Bell commercial in which basketball star Shaquille O'Neal is engulfed in flames, whereupon he calmly asks the camera "Is it hot in here, or is it just me?" Paralleling Barrett's work, the thesis that humans possess evolved psychological mechanisms dedicated to the acquisition of fire-relevant information predicts that, despite such misinformation and lack of experience, children in modern societies should resemble children in small-scale societies, where such resemblance occurs not with respect to fire knowledge per se, but rather with regard to the

1 Similar reasoning may explain the fascination with predators, both real and imaginary, that characterizes many modern cultures in which few individuals will ever encounter a truly dangerous animal (cf. Barrett, 2005). 
ease and rapidity with which they learn which substances burn and which do not, their tendency to make category errors within rather than across these two classes, and so on. With this in mind, I encourage investigators to explore the psychology of fire in children of both modern and developing nations.

In addition to having consequences for research into the psychological foundations of fire learning, the evolutionarily novel nature of western children's interactions with fire has potentially important implications for public policy. Fire safety officials spend a substantial amount of time, energy, and money trying to educate children regarding the dangers of fire, yet existing evidence suggests that, at best, these efforts have only limited impact on children's actual behavior (cf. Cotterall, 2003). In modern societies, adult-initiated pedagogy such as this plays an important role in information transfer. However, teaching is far less significant in the learning processes that occur in many small-scale societies. Rather, children spend much of their time either on the sidelines, watching the skilled performance of locally-adaptive behaviors, or attempting to engage in play-like learning behaviors (Fiske, n.d.; Mead, 1943); the same appears to be true of fire learning. It is possible that the latter mode is more appropriate for learning skills having a deep evolutionary history. The thesis that fire learning is shaped by a domain-specific information-acquisition mechanism suggests that opportunities to observe others interacting with fire in a mundane fashion, and opportunities to manipulate fire free of the titillation of proscription, may ultimately prove more effective than direct pedagogy in managing the threat to public safety posed by children's fire play in modern societies.

As noted earlier, again motivated by public safety concerns, a significant portion of the existing research on the psychology of fire focuses on aberrant individuals, persons who are at high risk of recidivism in the commission of fire-related crimes. Importantly, investigations of psychopathology may provide an additional avenue for exploring the evolved psychology of fire use. Just as phobias offer a window into the psychological mechanisms that evolved to cope with recurrent threats to ancestral humans (Öhman \& Mineka, 2001), so too may it be possible to explore manias as a window into the mechanisms that evolved to cope with recurrent resources or opportunities (examples unrelated to fire include erotomania and kleptomania). Considerable attention has been 
dedicated to the clinical study of pyromania. However, to date, investigators have largely framed the study of disordered fascination with fire using superordinate categories (e.g., impulse control disorders, aggressivity disorders, etc.) that overlook the unique importance of fire in human evolution. An evolutionary perspective suggests that useful insights into the mind of the pyromaniac may be gained by focusing on the specific features of fire that motivate fire-setting behavior; at present, we know little about how or why fire excites or stimulates such individuals. Likewise, light may be shed on pyromania by the thesis that, viewed both crossculturally and evolutionarily, western societies structure children's interactions with fire in a highly unusual manner - the ontogeny of this condition may epitomize the costs of disrupting the normal trajectory of information-acquisition processes that are guided by evolved mechanisms.

Alone among the components of the tool kits of our ancestors, fire continues to play important roles in contemporary human life. Whether as a useful tool or a destructive object of fascination, fire has an impact on societies large and small, yet we know little about the psychology of fire, fire learning, and the impact of culture on these factors. It is high time that we knew more.

\section{Appendix}

Survey Regarding Children's Interactions With Fire

1. Name of group/society/people at issue.

2. Do you know of any serious burns (including deaths) due to fire? Please briefly explain (i.e., age of individual[s], outcome, etc.).

3. At approximately what age do children first begin interacting with (i.e., manipulating, poking at, etc.) fire? Is there a gender difference in this regard?

4. At what age do children typically begin playing with fire (carrying burning objects, building play fires, etc.)? In what age range is such behavior most frequent, if it occurs? Any gender differences?

5. At what age are children fully competent to maintain a fire? At what age are children fully competent to start/build a fire? Any gender differences? 
6. Do children display entertainment interest in fire (i.e., watching fire for long periods, manipulating fires to create interesting effects, etc.)? Are there are age or gender differences? Do adults display such interest?

7. Do adults or older children directly instruct children with regard to the dangers of fire? How do children learn techniques for maintaining a fire? How do children learn techniques for starting/building a fire?

\section{REFERENCES}

Barrett, H. C.

2005 Adaptations to predators and prey. In D. M. Buss (Ed.), The evolutionary psychology handbook (pp. 200-223). Hoboken, NJ: Wiley.

Barrett, H. C., \& Behne, T.

2005 Children's understanding of death as the cessation of agency: a test using sleep versus death. Cognition, 96(2), 93-108.

Brain, C. K., \& Sillen, A.

1988 Evidence from the Swartkrans cave for the earliest use of fire. Nature, 336(6198),

Brown, D. E. 464-466.

1991 Human universals. New York: McGraw-Hill.

Clark, J. D., \& Harris, J. W. K.

1985 Fire and its roles in early hominid lifeways. African Archaeological Review, 3(1), $3-27$.

Cotterall, A. J.

2003 A survey of the disposition, affinity, interest and experience with fire of students in the city of Greater Sudbury. Unpublished M.A. thesis, Laurentian University, Sudbury, Ontario.

Curri, T. B., Palmieri, T. L., Aoki, T. H., Kaulkin, C. K., Lunn, M. E., Gregory, C. M., et al.

2003 Playing with fire: Images of fire on toy packaging. Fournal of Burn Care $\mathcal{E}^{\circ}$ Rehabilitation, 24(3), 163-165.

Fiske, A. P. Learning a culture the way informants do: observing, imitating, and participating.

(n.d.). Unpublished manuscript.

Garcia, J., Ervin, F. R., \& Koelling, R. A.

1966 Learning with prolonged delay of reinforcement. Psychonomic Science, 5, 121122.

Goren-Inbar, N., Alperson, N., Kislev, M. E., Simchoni, O., Melamed, Y., Ben-Nun, A., ET AL.

2004 Evidence of hominin control of fire at Gesher Benot Ya'aqov, Israel. Science, 304(5671), 725-727. 
Hall, J. R. J.

2003 Children playing with fire. Quincy, MA: National Fire Protection Association. Hardesty, V. A., \& Gayton, W. F.

2002 The problem of children and fire: An historical perspective. In D. J. Kolko (Ed.), Handbook on firesetting children and youth (pp. 1-13). San Diego: Academic Press.

KAFry, D.

1980 Playing with matches: Children and fire. In D. Canter (Ed.), Fires and human behavior (1st ed., pp. 47-61). New York: John Wiley \& Sons.

Kaplan, H., Hill, K., Lancaster, J., \& Hurtado, A. M.

2000 A theory of human life history evolution: Diet, intelligence, and longevity. Evolutionary Anthropology, 9(4), 156-185.

Kolko, D. J. (ED.).

2002 Handbook on firesetting: Children and youth. San Diego: Academic Press.

Kolko, D. J., Day, B. T., Bridge, J. A., \& Kazdin, A. E.

2001 Two-year prediction of children's firesetting in clinically referred and nonreferred samples. Fournal of Child Psychology \& Psychiatry \& Allied Disciplines, 42(3), 371-380.

MEAD, M.

1943 Our educational emphases in primitive perspective. American Fournal of Sociology, 48(6), 633-639.

Mineka, S., Davidson, M., Cook, M., \& Keir, R.

1984 Observational conditioning of snake fear in rhesus monkeys. Fournal of Abnormal Psychology, 93(4), 355-372.

ÖHMan, A.

1993 Stimulus prepotency and fear learning: Data and theory. In N. Birbaumer \& A. Öhman (Eds.), The organization of emotion: Cognitive, clinical and psychophysiological aspects (pp. 218-239). Toronto: Hogrefe and Hube.

Öhman, A., \& Mineka, S.

2001 Fears, phobias, and preparedness: Toward an evolved module of fear and fear learning. Psychological Review, 108(3), 483-522.

Okulitah, J. S., \& Pinsonneault, I.

2002 The interdisciplinary approach to juvenile firesetting: A dialogue. In D. J. Kolko (Ed.), Handbook on firesetting in children and youth (pp. 58-74). San Diego: Academic Press.

Palmieri, T. L., Aoki, T., Combs, E., Curri, T., Garma, S., Kaulkin, C., et al.

2004 Saturday-morning television: Do sponsors promote high-risk behavior for burn injury? Journal of Burn Care \& Rehabilitation, 25(4), 381-385.

Perrin-Wallqvist, R., Archer, T., \& Norlander, T.

2004 Adolescents' fire-setting awareness under boredom: Relation to personality variables. Psychological Reports, 94, 863-871.

Perrin-Wallqvist, R., \& Norlander, T.

2003 Firesetting and playing with fire during childhood and adolescence: Interview studies of 18-year old male draftees and of 18-19-year old female pupils. Legal and Criminological Psychology, 8(2), 151-157. 
Pinsonneault, I. L.

2002 Fire safety education and skills training. In D. J. Kolko (Ed.), Handbook on firesetting in children and youth (pp. 219-260). San Diego: Academic Press.

RAGIR, S.

2000 Diet and food preparation: Rethinking early hominid behavior. Evolutionary Anthropology, 9(4), 153-155.

Simonsen, B., \& Bullis, M.

2001 Fire interest survey: Final report. Salem, OR: Oregon Office of the State Fire Marshal.

Stanford, C. B.

1999 The hunting apes: Meat eating and the origins of human behavior. Princeton, N.J.: Princeton University Press.

Steen, F. F., \& Owens, S.

2001 Evolution's pedagogy: An adaptationist model of pretense and entertainment. fournal of Cognition and Culture, 1(4), 289-321.

Tooby, J., Cosmides, L., \& Barrett, H. C.

2005 Resolving the debate on innate ideas: Learnability constraints and the evolved interpenetration of motivational and conceptual functions. In P. Carruthers, S. Laurence \& S. Stich (Eds.), The innate mind: Structure and contents (pp. 305337). New York: Oxford University Press.

Wolf, A. P.

1993 Westermarck redivivus. Annual Review of Anthropology, 22, 157-175.

Wrangham, R. W., Jones, J. H., Laden, G., Pilbeam, D., \& Conklin-Brittain, N.

1999 The raw and the stolen: Cooking and the ecology of human origins. Current Anthropology, 40(5), 567-594. 